\title{
A Hybrid Chemical Based Metaheuristic Approach for a Vaccine Cold Chain Network
}

\author{
Kanon Sujaree \\ School of Industrial Engineering, Faculty of Engineering, \\ The innovation Center of Logistics and Water Transportation, \\ Rajamangala University of Technology Rattanakosin, Thailand \\ Email: kanon.suj@rmutr.ac.th (Corresponding Author) \\ Nara Samattapapong \\ School of Industrial Engineering, Institute of Engineering, \\ Suranaree University of Technology, Thailand \\ Email: nara@sut.ac.th
}

\begin{abstract}
One year ago, the coronavirus (COVID-19) virus began spreading around the world. Vaccines have now been developed by a variety companies from different countries. Vaccines must be stored in good condition within cold boxes designed to keep vaccines within acceptable temperature ranges. This paper proposes a hybrid algorithm based on a chemical reaction called Hybrid Artificial Chemical Reaction Optimization Algorithm (HACROA) which has been used to design a vaccine cold chain network in northern Thailand. The scope of this research is to focus on the Office of Disease Prevention and Control Area 1 (Chiang Mai, Thailand). The objective of this research is to rearrange routes to minimize total travel distances. Experiments were conducted to compare the efficiency of the hybrid algorithm with other algorithms in term of the distance. The optimal parameters of the algorithm employed a statistical experiment theory namely full factorial design. Experiment design and analysis were adopted to investigate the factors that affect the performance of this algorithm. HACROA was able to rearrange routes and found a better solution than the other algorithms.
\end{abstract}

Keywords: vaccine, hybrid artificial chemical reaction optimization algorithm, experimental design

\section{INTRODUCTION}

In late December 2019, a previous unidentified coronavirus, now named the 2019 novel coronavirus, emerged in Wuhan, China before spreading around the world. The disease was officially named as Coronavirus Disease-2019 (COVID-19) by The World Health Organization (WHO) on February 11, 2020 (Yi-Chi Wua et al., 2020). The COVID-19 pandemic has massively disrupted supply chains at the global and local scales (Pujawan and Bah, 2021). Many countries consequently undertook research to find a vaccine for COVID 19, which can be classified into following four categories (Peiwen Zhou et al., 2020).

1. Inactivated vaccine is the most likely type of vaccine. It is owned by Sinovac, Sinopharm, Wuhan Institute.
This vaccine is produced with difficulty even without the use of new technology and it is not possible to be produced quickly. It is a type of vaccine that is likely to be effective because it kills the whole virus. There are subsequently no concerns and it is a technology that has previously been used in the manufacture of other vaccines. The vaccine was expected to hit the market the fastest, but it is likely to be the most expensive. This group of vaccines must be stored at temperatures between $2-8^{\circ} \mathrm{C}$

2. Adenovirus Vector vaccine includes the gene for the virus that causes COVID-19. Notable adenoviruses include China's CanSino, which uses human adenovirus strains, the British company Astra Zeneca in collaboration with Oxford University which uses, a chimpanzee virus, and Russia's Gamaleya. These vaccines have now started being used in vaccination programs. If an individual has never been infected with the adenovirus strain that is taken as a carrier (vector) before, there are disadvantages. First, the adenovirus itself is a live virus vector and when injected, it can cause serious problems. Therefore, these types of vaccines are not suitable for people with an immunodeficiency. This group of vaccines must be stored at $2-8^{\circ} \mathrm{C}$.

3. mRNA vaccines, notably Moderna, is sponsored by the US National Institute of Health. Certain countries have now approved Moderna's vaccine which was found to prompt a strong immune response among in normal people, including the elderly. Many companies are producing similar vaccines, such as Germany's BioTech + Pfizer. This group of vaccines must be stored at $-70^{\circ} \mathrm{C}$. This type of vaccine uses a new technology that has never been used in humans before and has the advantage of being quick and easy to produce. Nonetheless, since this technology has not been used previously, there are concerns that although there were no problems with the side effects from shortterm studies, there is no research available to support long-term side effects. 
4. Protein-base vaccine using virus shell protein production technology. This is a well-known technology because it is used in other vaccines such as influenza, hepatitis $\mathrm{B}$, and so on with an immunestimulating adjuvant. This type of vaccine is fast to produce. The most prominent company developing this type of vaccine is Novavax of America which is now entering Phase III research. Sanofi is partnering with GSK, two of the biggest companies in the industry and uses, using technology similar to other vaccines. This group of vaccines must be stored at $2-8^{\circ} \mathrm{C}$.

The WHO is serious about the importance of transportation which it sometimes refers to as the immunization supply chain. According to WHO the purpose of Vaccine Cold Chain (VCC) is to maintain vaccine quality from the time of manufacture until the point of administration by ensuring that vaccines are stored and transported within acceptable temperature ranges. Vaccines are highly useful for the treatment and prevention of diseases in both children and adults. However, the effectiveness of vaccines depends on appropriate storage conditions and transportation which can be difficult to control. Vaccines are sensitive biological products. Some vaccines are sensitive to freezing, some to heat and others to light (World Health Organization, 2015). In Thailand, VCC has been started by international vaccine manufacturers for vaccines to be delivered by aircraft. Both domestic and overseas vaccines are shipped to the Department of Disease Control (DDC) before being distributed from there using refrigerated vehicles to all 13 Offices of Disease Prevention and Control (ODPC) throughout the country. Each ODPC delivers vaccines to the Province Health Office (PHO). Throughout the supply chain, the vaccines are shipped from $\mathrm{PHO}$ to hospitals or Contracting Units for Primary Care (CUP). The Primary Care Centre requires vaccinations from the CUP in the last chain (Dechatawat, 2012). Vaccines are currently delivered by ODPC to PHO and are transported to CUP. Each ODPC transports the vaccines to the areas within their own area of responsibility. ODPC 1 is responsible for Chiang Mai, Chiang Rai, Nan, Phayao, Mae Hong Son, Lamphun, Lampang and Phrae (The ODPC 1 in Thailand, 2017). The vaccines will be transported to 102 public hospitals within ODPC 1. The importance of this research is to reduce transportation time and ensure the effectiveness of vaccines since they must be transported at controlled temperatures. Thailand's northern region is mountainous which can slow road transportation. A simple vaccine cold chain network is described in Figure 1. ODPC 1 generally uses one refrigerated vehicle to support each $\mathrm{PHO}$. The same format is used for the delivery of the COVID-19 vaccine. In the first quarter of 2021, Thailand will begin the first phase 2 million doses of the inactivated vaccine and 26 million doses of the adenovirus Vector vaccine. Google Maps is used to calculate the distances between ODPC 1, PHOs and CUPs. The distance information between the PHOs and CUPs is stored in the form of a symmetric matrix.

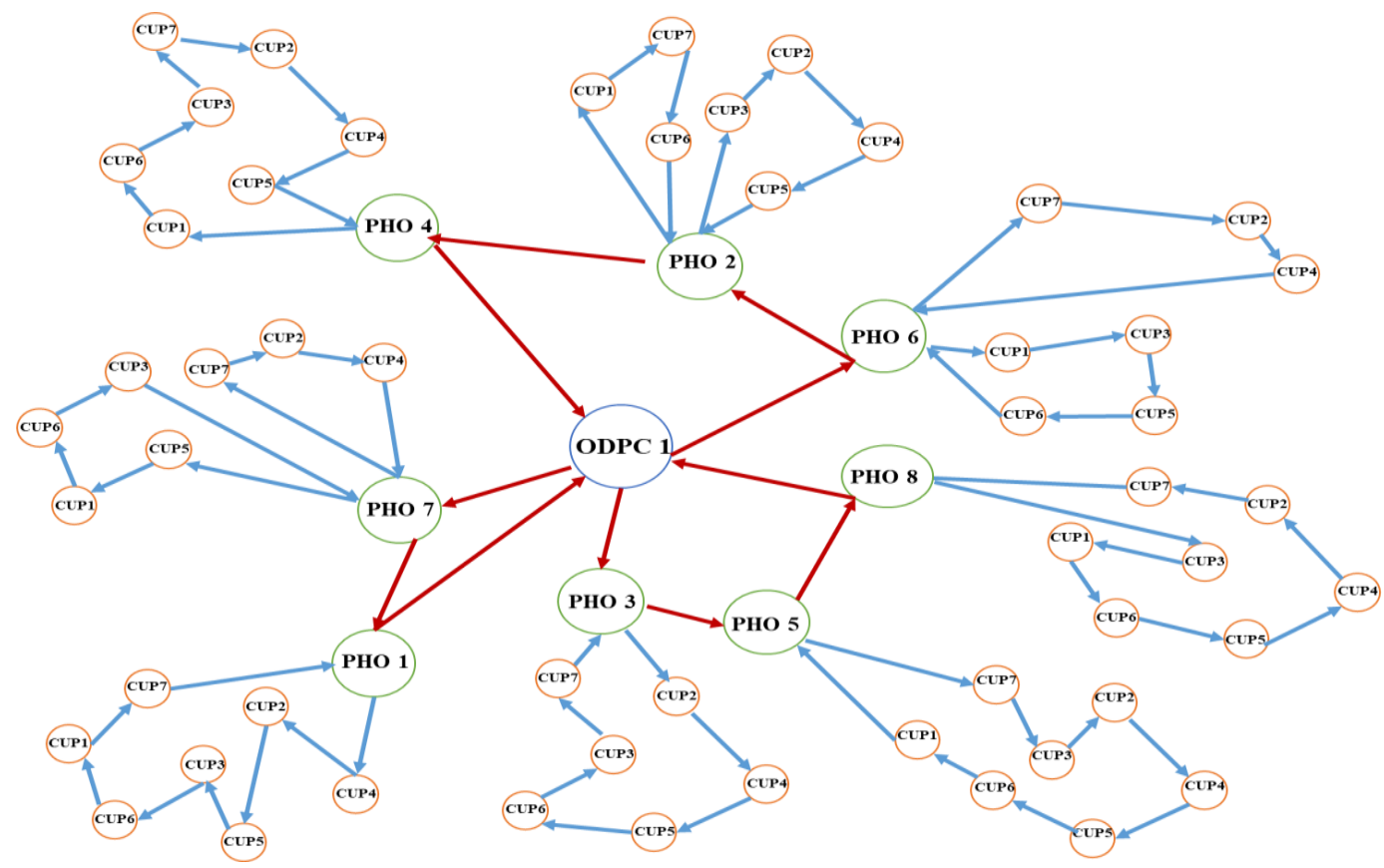

Figure 1 A simple vaccine cold chain network. The refrigerated vehicle starts from ODPC 1 and delivers to each PHO and returns to ODPC 1. (Note: The refrigerated vehicle of each PHO delivers to the CUP member in its area of responsibility and finishes at the PHO. The capacity of refrigerated vehicle must not be exceeded).

During recent years, research in applying machine learning (ML) to design efficient, effective and robust metaheuristics has become increasingly popular (El-Ghazali, 2020; Haydar et al., 2020; Wecka et al, 2020). Machine learning algorithms could provide better accuracy and less computational cost for demand forecasting than traditional forecasting models (Ammar, 2021). Metaheuristic approaches are considered for large-scale optimization problems. Typical metaheuristic optimization approaches are commonly physics-based, biology-based, chemistrybased, sociology-based and ethnomusicology based (Sujaree, 2017). Many of the proposed versions are 
population-based techniques, in which they begin searching for a solution with multiple points such as genetic algorithm (Goldberg, 1989), particle swarm optimization(Kennedy and Eberhart, 1995), harmony search algorithm (Lee and Geem, 2005), cuckoo search algorithm (Xin and Suash, 2010), and artificial carbon nanotube synthesis optimization (Sujaree, 2020). Many proposed methods are random based techniques such as ant colony optimization (Dorigo and Maziezzo, 1996), and central force optimization (Formato, 2007). This paper proposes a metaheuristic approach called Hybrid Artificial Chemical Reaction Optimization Algorithm (HACROA) which has been used to minimized the distance from ODPC 1 to CUPs in the area of responsibility. HACROA was inspired by the chemical reaction theory and was developed from the Artificial Chemical Reaction Optimization Algorithm (ACROA) (Alatas, 2012).

\section{FORMULATIONS AND PROBLEM STATEMENT}

The vaccine cold chain network is a type of classical Vehicle Routing Problem (VRP) also known as the capacitated VRP. This VRP seeks to design optimal routes where each vehicle travels a single route from a single central depot. The objective of the VRP is to find a dataset of the least total cost or distance. Only one vehicle visits each customer, the vehicle starts its routes from the depot and then ends at the same depot. However, the delivery must not exceed the vehicle's capacity (Jaegere et al., 2016). Several variants of VRP have been studied to address the wide variety of conditions. For example, capacitated VRP (CVRP) (Ralhs et al., 2003), multi depot VRP (MDVRP) (Lysgaard et al., 2004), VRP with time windows (VRPTW) (Solomon, 1987), heterogeneous fleet VRP (HVRP) (Baldacci et al., 2008), and VRP with simultaneous pickup and deliveries (VRPSPD) (Dethloff, 2001). Real-time VRP on large road networks with time-dependent travel time remains a challenge due to the required computation time for optimal vehicle routes with more delivery points or routes which significantly increases in line with the size of the road network (Chen, 2018).

The mathematical model and notations for the vaccine cold chain network considered in this paper utilize the following equations.

Indices:

$\begin{array}{lll}\text { - } & Z & \text { denotes total distance } \\ \text { - } & i, & \text { denotes OPDC } i \\ \text { - } & j & \text { denotes PHO } j \\ \text { - } & m & \text { denotes CUP } m \\ \text { - } & k & \text { denotes refrigerator vehicles } k \text { from ODPC to PHO } \\ \text { - } & l & \text { denotes refrigerator vehicles } l \text { from PHO to CUP } \\ \text { - } & p & \text { is PHO }(1,2,3, \ldots \ldots, \mathrm{N}) \\ \text { - } & q & \text { is CUP }(1,2,3, \ldots \ldots, \mathrm{M}) \\ \text { - } & c & \text { is the capacity of refrigerator vehicles } \\ \text { - } & a & \text { is demand } \\ \text { Parameters: }\end{array}$

- $\quad d_{i j}$ is distance from OPDC $i$ to $\mathrm{PHO} j$

- $\quad d_{j m}$ is distance from PHO $j$ to CUP $m$

- $\quad N$ is number of PHO

- $M$ is number of CUP

- $K$ is number of vehicles from ODPC1 to PHO

- $L$ is number of vehicles from PHO to CUP
- $\quad v \quad$ is vaccine from ODPC1 to PHO

- $y$ is vaccine from PHO to CUP

\section{Decision variables:}

- $\quad X_{i j}^{k}=1$ if vehicles $k$ from ODPC $i$ to $\mathrm{PHO} j$, otherwise 0

- $\quad X_{j m}^{l}=1$ if vehicles $l$ from PHO $j$ to CUP $m$, otherwise 0

- $\quad Y_{i}^{k}=1$ if vaccines load in vehicles $k$, otherwise 0

- $Y_{j}^{l}=1$ if vaccines load in vehicles $l$, otherwise 0

- $U_{i},=$ Auxiliary variable $\geq 0$

- $U_{j},=$ Auxiliary variable $\geq 0$

- $U_{m}=$ Auxiliary variable $\geq 0$ Objective function

$\operatorname{MinZ}=\sum_{i=1}^{N} \sum_{j=1}^{N} \sum_{k=1}^{K} d_{i j} X_{i j}^{k}+\sum_{j=1}^{N} \sum_{j=1}^{M} \sum_{m=1}^{M} \sum_{l=1}^{L} d_{j m} X_{j m}^{l}$

Representation by

$$
\begin{aligned}
& \sum_{j=1}^{N} X_{i j}^{k} \leq 1 \quad(k=1,2,3, \ldots \ldots \ldots K) \\
& \sum_{m=1}^{M} X_{j m}^{l} \leq 1 \quad(l=1,2,3, \ldots \ldots . . L) \\
& \sum_{i=0}^{N} X_{i p}^{k}-\sum_{j=0}^{N} X_{p j}^{k}=0(p=1 \ldots N) \\
& \sum_{j=0}^{M} X_{j q}^{l}-\sum_{m=0}^{M} X_{j q}^{l}=0(q=1 \ldots M) \\
& \sum_{k=1}^{K} Y_{i}^{k}=1 \quad(i=1,2,3 \ldots K) \\
& \sum_{l=1}^{L} Y_{j}^{l}=1 \quad(j=1,2,3 \ldots L) \\
& \sum_{i=1}^{N} c_{i}^{v} Y_{i}^{K} \leq a_{k}^{v} \quad(k=1 \ldots K) \\
& \sum_{j=1}^{M} c_{j}^{y} Y_{j}^{L} \leq a_{L}^{y}(l=1 \ldots . L) \\
& Y_{i}^{k} \leq \sum_{i=1}^{N} X_{i j}^{k}(i=1 \ldots . . N),(k=1 \ldots . K) \\
& Y_{j}^{l} \leq \sum_{j=1}^{M} X_{j m}^{l}(j=1 \ldots M),(l=1 \ldots . L) \\
& \sum_{k=1}^{K} \sum_{i=0}^{N} X_{i j}^{k} \geq 1 \quad(j=1,2,3 \ldots . . N) \\
& \sum_{l=1}^{L} \sum_{j=1}^{M} X_{j m}^{l} \geq 1 \quad(m=1,2,3 \ldots . M) \\
& U_{i}-U_{j}+N \sum_{k=1}^{K} X_{i j}^{k} \leq N-1, \forall_{i, j}=(2, \ldots . . N), i \neq j \\
& U_{j}-U_{m}+N \sum_{l=1}^{L} X_{j m}^{l} \leq N-1, \forall_{j, m}=(2, \ldots . N), j \neq m
\end{aligned}
$$

Equation 1 is a sum of the distance from ODPC1 to the PHOs. Constraints 2 and 3 represent the refrigerated vehicles from ODPC1 to the PHOs and the PHOs to the CUPs, respectively. Constraints 4 and 5 determine that the vaccines are only delivered when required. Likewise, both constraints 6 and 7 indicate that only refrigerated vehicles are delivered 
to the PHOs and CUPs. Constraints 8 and 9 ensure that the transported vaccines are below or equal to the capacity limitation of the refrigerated vehicles in the PHOs and CUPs network. To ensure the refrigerated vehicles $k$ and $l$ take a route through PHO $j$ and CUP $m$ by constraints 10 and 11 .

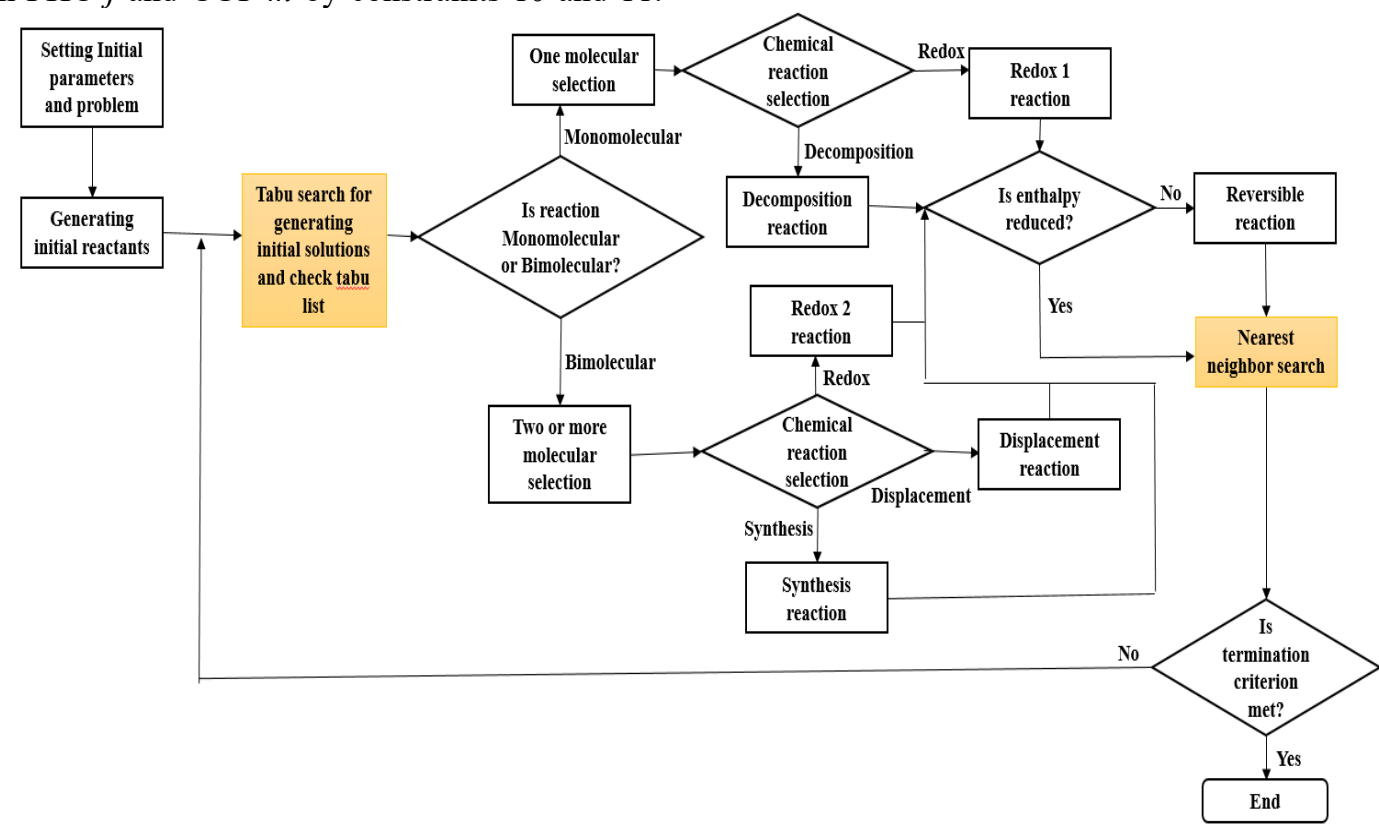

Figure 2 Flowchart of the Hybrid Artificial Chemical Reaction Optimization Algorithm (HACROA) for the vaccine cold chain network

The chemical reaction can be classified into seven reactions including synthesis, decomposition single displacement, souble displacement, combustion, redox and
At least one time, both PHO $j$ and CUP $m$ get vaccinations which is ensured by constraints 12 and 13. The remaining constraints prevent the sub-tour in PHO and CUP network respectively.

\section{HACROA FOR THE VACCINE
COLD CHAIN NETWORK \\ 3. HACROA FOR THE VACCINE
COLD CHAIN NETWORK PROBLEM}

reversible. Both tabu search and nearest neighbour search were inserted into the initial solution and final solution (local search in offspring), respectively as shown in Figure 2. The initial process involves setting parameters for both the algorithm and the problem. The second step is to define the number of iteration, reactants, and random integer (CUPs) into the string. The reactant is in the form of an asymmetric matrix and shown in Figure 3.

\section{CUPs}

\begin{tabular}{|c|c|c|c|c|c|c|c|c|c|}
\hline PHO1 & 3 & 4 & 1 & 2 & 6 & 8 & 9 & 5 & 7 \\
\hline $\mathrm{PHO}_{2}$ & 2 & 6 & 1 & 7 & 5 & 3 & 4 & & \\
\hline PHO3 & 3 & 5 & 7 & 8 & 1 & 4 & 2 & 6 & \\
\hline PHO4 & 4 & 6 & 9 & 7 & 8 & 1 & 2 & 3 & 5 \\
\hline PHO5 & 5 & 4 & 3 & 1 & 2 & & & & \\
\hline PHO6 & 5 & 6 & 1 & 4 & 3 & 2 & & & \\
\hline PHO7 & 7 & 5 & 1 & 6 & 2 & 3 & 4 & & \\
\hline PHO8 & 4 & 6 & 7 & 2 & 5 & 1 & 3 & & \\
\hline
\end{tabular}

Figure 3 The asymmetric matrix represents the reactant that shows the delivery of the vaccine from the PHO to CUPs to calculate the objective function in Equation 1. 
The next process, the application seeks to evaluate the objective function from Equation 1. The third step is a random number between 0 and 1 . Monomolecular reactions require one reactant string. There are two reactants namely redox 1 and decomposition in the molecular reactant. Decomposition reactant: Two random points in the reactant string are selected and the CUPs between those points are reversed. Redox 1 reactant: A new random reactant string compares the objective function and chooses a better solution for the next process. The bimolecular reactants are redox 2 , displacement and synthesis. This type of reaction requires two reactant string participating. Redox 2 reactants: Two points are randomly selected and the numbers (CUPs) of reactants between the two points are exchanged similar to the two 2-point crossover used in the genetic algorithm. Displacement reaction: In this research, swapping based on randomness is used to exchange the numbers (CUPs) between two reactants. Synthesis reaction: Non-matching numbers (CUPs) of two reactants are the new random. The fourth step is to check that the condition of the enthalpy process is reduced. A reversible reaction involves swapping the first point with the last point of the reaction string. The HACROA is terminated when the termination criterion has been met. The application reports the best solution. Otherwise, the algorithm is repeated. The roots of tabu search go back to the 1970's, and Glover was the first researcher to present this approach for the optimization problem (Glover, 1986). In this paper, Tabu search is used to check the new solution and is compared with the offspring solution in the tabu list. This process is used to prevent duplicate routes and to distribute the solution to cover the problem area. Good solutions are inserted and poor solutions are eliminated. Nearest neighbour search is an example of an optimization problem, in which the goal is to find a point which minimizes certain objective function (Knuth, 1973). This approach is used to improve the objective function of the offspring in the final process. The HACROA for the vaccine cold chain network problem is shown in Figure 4.

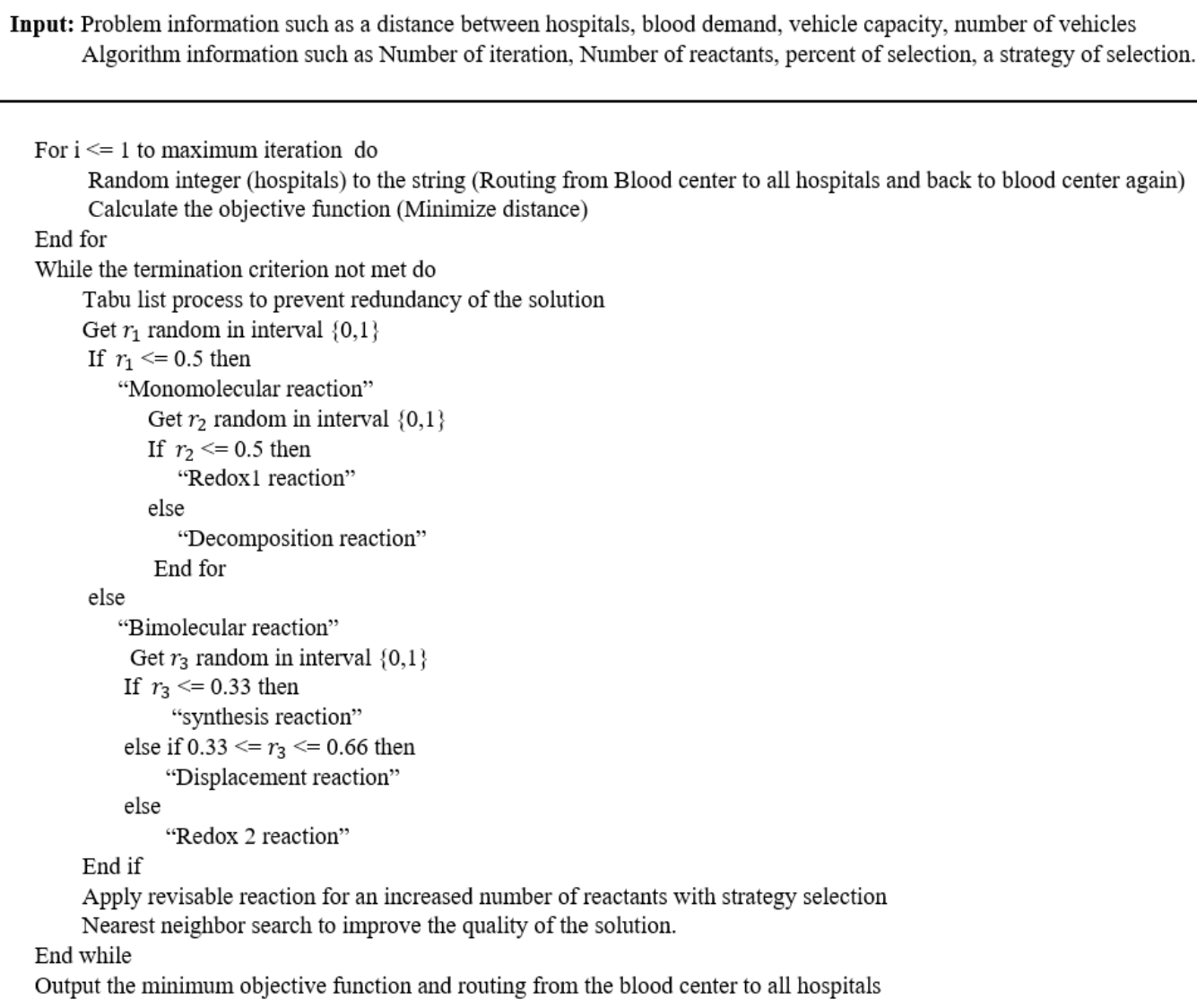

Apply revisable reaction for an increased number of reactants with strategy selection

Nearest neighbor search to improve the quality of the solution.

Figure 4 The pseudocode of HACROA for the vaccine cold chain network

\section{EXPERIMENTAL DESIGN}

Currently, there are several factors impacted by these algorithms. In the present work, the statistic of experiment theory was used to design an experiment 3 level of full factorial design. This approach was applied when there were $\mathrm{k}$ factors to consider. Each factor consists of three levels, High, Medium, and Low. One experiment composed of $3^{\mathrm{k}}$ data and is called $3^{\mathrm{k}}$ factorial design. The application was developed in visual basic trial version and was calculated on a PC with Intel Core i7 $3.40 \mathrm{GHz}$ and RAM 8 GB. Design of the experiment was evaluated in a Minitab 18.1 trial version for 30 days. A two-step sequential experiment was adopted in this work. The first experiment was designed to investigate the appropriate setting for HACROA's parameters including the number of iterations $(i)$, the number of reactants $(r)$, the percentage of reaction $(p r)$, and the percentage of monomolecular $(\mathrm{pm})$. Since each parameter was considered at three levels (see Table 1), full factorial design $\left(3^{\mathrm{k}}\right)$ was adopted in this experiment (Montgomery, 2001). 
Table 1 Determining the factors and levels of the full factorial design experiment of HCROA.

\begin{tabular}{cccc}
\hline \multirow{2}{*}{ Factors } & \multicolumn{3}{c}{ Levels } \\
\cline { 2 - 4 } & Low & Medium & High \\
\hline$i^{\star} r$ & $50 / 200$ & $100 / 100$ & $200 / 50$ \\
$p r$ & $25 \%$ & $50 \%$ & $75 \%$ \\
$p m$ & $25 \%$ & $50 \%$ & $75 \%$
\end{tabular}

Higher values of these parameters indicate a greater chance of getting good solutions, although this requires more computational time. The combination of these factors was fixed to 10,000 candidate solutions in order to make a reasonable comparison by determining the number of candidate solutions. The probability of a solution is around 822,360 solutions. The experiment was repeated five times using different random seed numbers, which could be a potential nuisance factor. The computational results obtained from 135 runs shown in Table 2 were analyzed using a general linear form of analysis of variance (ANOVA) which included Source of Variation, Degree of Freedom (DF), Sum of Square(SS), Mean Square(MS), F values and P values. A factor with $\mathrm{p}<=0.05$ is statistically significant at a $95 \%$ confidence level.

Table 2 ANOVA results obtained from HACROA by analysis factors affecting the solution.

\begin{tabular}{|c|c|c|c|c|c|}
\hline Source & DF & $\mathrm{SS}$ & MS & $\mathrm{F}$ & $\mathrm{P}$ \\
\hline$i * r$ & 2 & 58061 & 29030 & 1.99 & 0.142 \\
\hline$p r$ & 2 & 342706 & 17135 & 11.75 & 0.000 \\
\hline$p m$ & 2 & 1263 & 631 & 0.04 & 0.958 \\
\hline$(i * r) *(p r)$ & 4 & 28297 & 7074 & 0.48 & 0.747 \\
\hline$(i * r) *(p m)$ & 4 & 69470 & 17367 & 1.19 & 0.319 \\
\hline$(p r) *(p m)$ & 4 & 18031 & 4508 & 0.31 & 0.871 \\
\hline$(i * r) * p r * p m$ & 8 & 176221 & 22028 & 1.51 & 0.163 \\
\hline Error & 108 & 1517020 & 14587 & & \\
\hline Total & 134 & 2269896 & & & \\
\hline
\end{tabular}

In Figure 5, the residual plot represents the distribution pattern of distance data to show the normal probability and a histogram plot. Versus fits graphs and versus order graphs show the data distribution and all variables are independent.

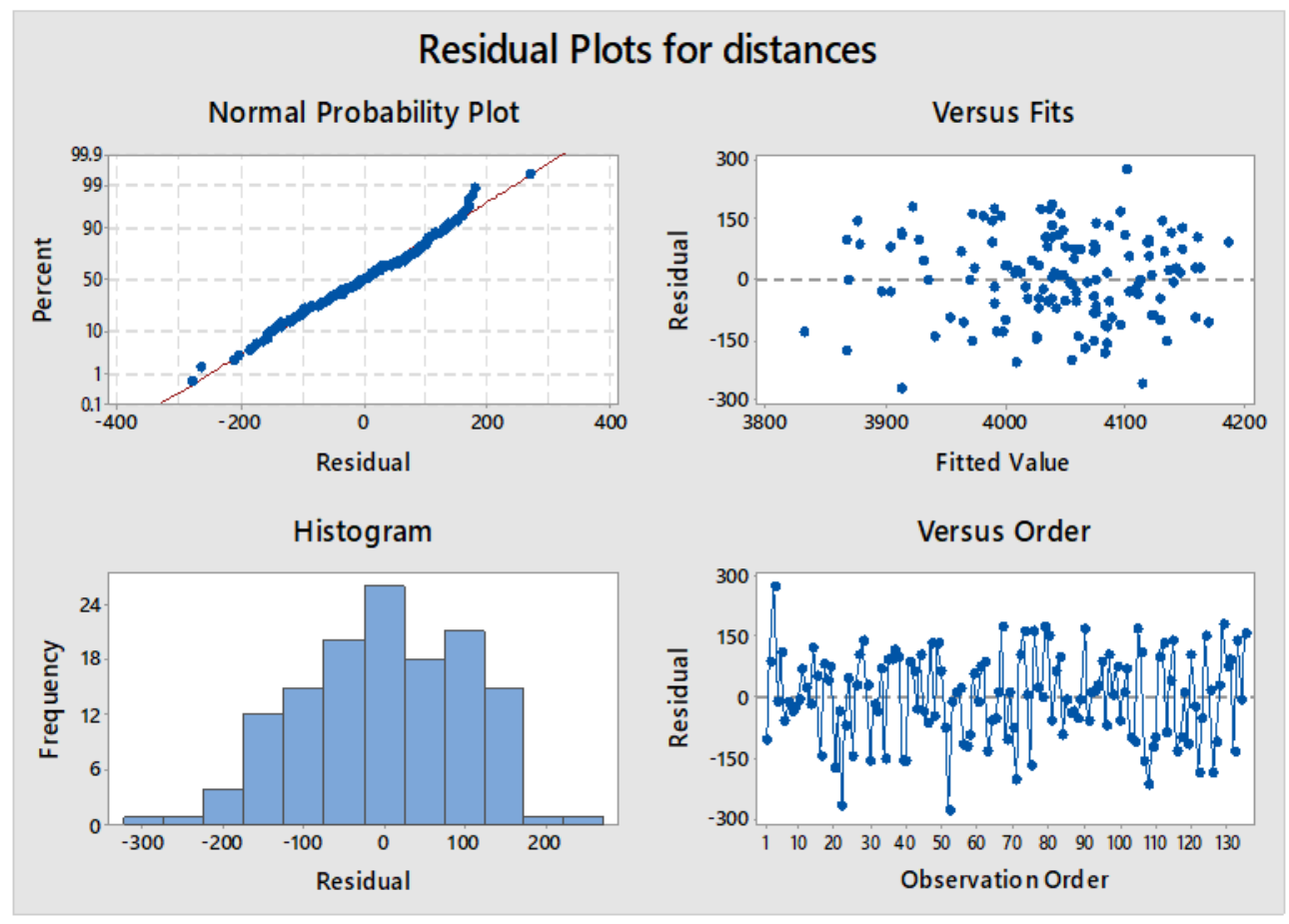

Figure 5 Residual plot demonstrating the data distribution of data and the independence of variables. This graph consists of 4 plots including normal probability, histogram, versus fits, and versus order 
The main effect plots in Figure 5 represent the appropriate setting of HACROA's parameters $\left(i^{*} r, p r, p m\right)$ should be $200 / 50,75 \%$ and $75 \%$ respectively. Due to the mean of the main effect plot in Figure 6 is minimal average distance.

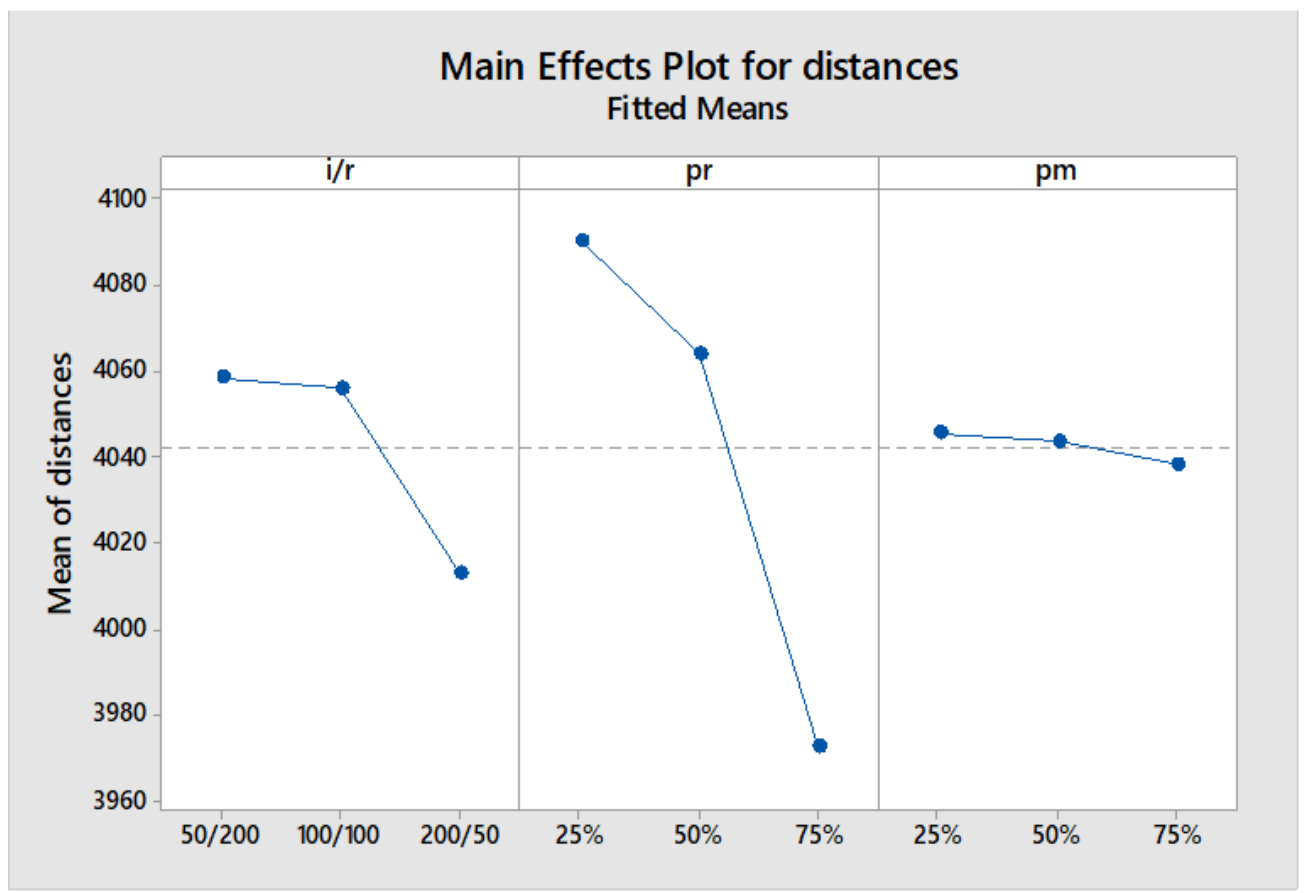

Figure 6 Main effect plots of HACROA which present an average distance of each factor, where pr gives the minimum solution of $75 \%$.

In this research, the proposed algorithm is compared with the results from previous publications including MaxMin Ant System (MMAS) (Sujaree, 2019), Central Force Optimization (CFO) (Sujaree, 2018), Hybrid Central Force Optimization (HCFO) (Sujaree, 2018) and Artificial
Chemical Reaction Optimization Algorithm (ACROA). The appropriate parameters from the full factorial design were processed 10 times to analyze route distance. These algorithms are based on the random technique. As a result, the route distances are not equal as shown in Table 3.

Table 3 Algorithms presenting the average calculation time and total distance from the appropriate parameters

\begin{tabular}{|c|c|c|c|c|c|c|c|c|}
\hline Times & MMAS & (22.77 sec.) & CFO & (21.36 sec.) & HCFO (24.65sec.) & ACROA (20.36 sec.) & HACROA & (23.48 sec.) \\
\hline 1 & & 3848 & & 3898 & 3848 & 3881 & & 3832 \\
\hline 2 & & 3867 & & 3872 & 3854 & 3872 & & 3849 \\
\hline 3 & & 3898 & & 3826 & 3867 & 3826 & & 3854 \\
\hline 4 & & 3826 & & 3854 & 3838 & 3854 & & 3872 \\
\hline 5 & & 3881 & & 3872 & 3848 & 3867 & & 3826 \\
\hline 6 & & 3838 & & 3906 & 3872 & 3848 & & 3804 \\
\hline 7 & & 3854 & & 3867 & 3804 & 3906 & & 3816 \\
\hline 8 & & 3826 & & 3854 & 3898 & 3838 & & 3898 \\
\hline 9 & & 3906 & & 3849 & 3804 & 3881 & & 3792 \\
\hline 10 & & 3854 & & 3881 & 3826 & 3898 & & 3814 \\
\hline
\end{tabular}

Note: The minimal distance of MMAS, CFO, HCFO, ACROA, and HACROA are 3826, 3826, 3804, 3826, and 3792 kilometers, respectively.

Vaccines are currently transported by the shortest path method, which means selecting CUPs to send to next based on the nearest distance. The total distance travelled from
ODPC 1 to all CUPs is approximately $3,923 \mathrm{~km}$. The summary of the objective function obtained from HACROA was able to determine a distance of $3,792 \mathrm{~km}$. Therefore, the 
best results obtained thus far from HACROA were better than the other algorithms.

\section{CONCLUSION}

This research proposed a hybrid algorithm called Hybrid Artificial Chemical Reaction Optimization Algorithm (HACROA) which was used to minimize the total distance within the vaccine cold chain network including the Office of Disease Prevention and Control 1 (ODPC 1), Province Health Office (PHO) and Contracting Unit for Primary care (CUP) in the area of responsibility. This paper could be applied to the delivery of the COVID-19 vaccines to preserve the properties of the vaccine in order to suppress the spread of the virus. This algorithm is implemented by improving local search solutions. Both tabu search and nearest neighbor search were used to enhance efficiency. The performance of an algorithm depends on its parameter settings. The experiment was based on a full factorial design aiming to investigate the appropriate setting of HACROA's parameters, which was then used in the experiment intended to compare the performance of the proposed method with the real system. The experiment showed that HACROA found a shorter route than the real system, MMAS, CFO, HCFO, and ACROA. However, there are many constraints for transportation. Some CUPs have sufficient vaccines but some CUPs lack vaccines. It is therefore recommended to check the vaccine stocks of CUPs in real time and enter the distance data to assess the application for more accurate processing.

\section{ACKNOWLEDGEMENTS}

This research was supported by the Faculty of Engineering, RMUTR. Thanks to information from the health data center.

\section{REFERENCES}

Alatas, B., (2012). A novel chemistry based metaheuristic optimization method for mining of classification rules, Expert Systems with Applications, 39(12), pp. 1108011088.

Ammar, A. M., (2021). Data Analytics in the Supply Chain Management: Review of Machine Learning Applications in Demand Forecasting, Operation and Supply Chain Management (OSCM): An International Journal, 14(1), pp. 1-13.

Baldacci, R., Vigo, D., \& Battarra, M., (2008). Routing a heterogeneous fleet of vehicle, Operations Research, (43), pp. 3-27.

Chen, X., (2018). An improved efficient algorithm for time dependent vehicle routing. Operation and Supply Chain Management (OSCM): An International Journal, 11(2), pp. 55-65.

Dechatawat, S., (2012). Vaccine cold chain system, https://scm.gpo.or.th/vmi/document/FluNHSO/07.pdf, Access date: 2 April 2018.

Dethloff, J., (2001). Vehicle routing and reverse logistics: The vehicle routing problem with simultaneous delivery and pick-up. OR-Spektrum, 23(1), pp.79-96.

Dorigo, M, Maziezzo, V., \& Colorni, A., (1996). The ant system: optimization by a colony of cooperating agents, In IEEE Transactions on Systems, Man, and Cybernetics, Part B, 26(1), pp. 29-41.

El-Ghazali, T., (2020). Machine learning into metaheuristics: A survey and taxonomy of data-driven metaheuristics.,https://hal.inria.fr/hal02745295/ document.

Formato, R.A., (2007). A new metaheuristic with applications in applied electromagnetics, Progress in electromagnetics research, (77), pp. 425-491.

Glover, F., (1986). Future paths for integer programming and link to artificial intelligence, $J$. computers and operation research, 13(5), pp.533-549.

Goldberg, D.E., (1989). Genetic Algorithm in Search Optimisation and Machine Learning, Addison- Wesley publishing company, Alabama.

Haydar, Y., Wanying, S., \& Zafrin, R., (2020). A Review and Scientometric Analysis of Supply Chain Management (SCM), Operation and Supply Chain Management (OSCM): An International Journal, 13(2), pp. 123-133.

Jaegere, N.D., Defraeye, M., \& Nieuwenhuyse L.V., (2016). The vehicle routing problem: state of the art classification and review, Computer \& industrial engineering, (99), pp. 300-313.

Kennedy, J., \& Eberhart, R.C., (1995). Particle swarm optimization, In Proc. of ICNN'95 - Int. Conf. on Neural Networks, ed. IEEE, Perth, WA, Australia, pp. 1942-1948.

Knuth, D., (1973), The Art of Com. Programming, Vol. 1-3 Addison-Wesley Longman Publishing Co., Inc., Boston.

Lee, K.S., \& Geem Z.W., (2005). A new meta-heuristic algorithm for continuous engineering optimization: Harmony search theory and practice, Comput. Methods Appl. Mech. Engrg. 194, pp. 3902-3933.

Lysgaard, J., Letchford, A.N., \& Eglese, R.W., (2004). A new branch-and-cut algorithm for the capacitated vehicle routing problem, Mathematical Programming, (100), pp. 423-445.

Montgomery, D.C., (2001). Design \& Experiment, John Wiley and Sons, New York.

Pujawan, I.N. \& Bah, A.U., (2021). Supply chains under COVID-19 disruptions: literature review and research agenda, Supply Chain Forum: An International Journal, https://doi.org/10.1080/16258312.2021.1932568

Ralhs, T.K., Kopman, I., Pulleyblank, W.R., \& Trotter, L.E., (2003). On the capacitated vehicle routing problem, Mathematical programming, (94), pp.343-359.

Solomon, M.M., (1987). Algorithm for Vehicle routing and scheduling problem with time window constraints, Operations Research, 35(2), pp. 254-265.

Sujaree, K., (2017). Blood vehicle routing network using artificial chemical reaction optimization algorithm, In Proc. 2nd Int. Conf. Times-con, ed. Mahidol university (Salaya, Nakhonpathom, Thailand, pp. 189-195.

Sujaree, K., (2018). A Physics based metaheuristic approach for vaccine cold chain network problem, In Proc. 4th Conf. Rajamangala manufacturing and management, ed. Rajamangala university of Technology Srivijaya (Deevana plaza, Krabi, Thailand), pp. 583-591. 
Sujaree, K., (2018). Vaccine cold chain network problem using hybrid central force optimization, CRMA Journal, (16), pp 63-76.

Sujaree, K., (2019). Designing a vaccine cold chain network in northern Thailand using the Max-Min ant system, Suranaree J. Sci. Technol, 26(3), pp. 257-265.

Sujaree, K., (2020). Artificial Carbon Nanotube Synthesis Optimization to Address the Vaccine Cold Chain Network Problem. International Journal of Modeling and Optimization, 10(1), pp. 32-40.

The ODPC 1 in Thailand, (2017), Area of responsibility and Heath service, http://odpc1.ddc.moph.go.th/area61.html, Access date: 10 April 2018.

Wecka, I. Y., Evri, M.R., I-soon, R., \& Vatanavongs, R., (2020). Exploring Big Data Research: A Review of Published Articles from 2010 to 2018 Related to
Logistics and Supply Chains, Operation and Supply Chain Management (OSCM): An International Journal, 13(2), pp. 134-149.

World Health Organization (WHO), Immunization, (2015). http://www.who.int/ immunization, Access date: 14 March 2018.

Xin, S.Y., \& Suash, D., (2010). Cuckoo search via Lévy flights, Int. J. Mathematical Modeling and Numerical Optimisation, 1(4), pp. 210-214.

Yi-Chi, W., Ching-Sung, C., \& Yu-Jiun, C., (2020). The outbreak of COVID-19: An overview, Journal of Chinese Medical Association, 83, pp. 217-220.

Zhou, Li, Xie, An, Fan, Wang, Yiwei Li, Liu, Wu, Geng Li, \& Qin Li, (2020), Research progress and challenges to coronavirus vaccine development, Journal of Medical Virology, 93(2), pp. 741-754.

Kanon Sujaree, Assistant Professor of Industrial Engineering in the Faculty of Engineering at Rajamangala University of Technology Rattanakosin, Thailand. Dr. Kanon graduated with a bachelor's degree in Industrial Engineering, Faculty of Engineering from Naresuan University Thailand (1999-2003), and with master's degrees in Engineering Management, Faculty of Engineering from Naresuan University Thailand (2004-2007), and Ph. D. in Nanoscience and Technology from Chulalongkorn University, Thailand (2009-2015). His research interests consist of meta-heuristics, optimization, industrial statistics, logistics, and supply chain systems.

Nara Samattapapong, Lecturer of the School of Industrial Engineering, Suranaree University of Technology, Thailand. Dr. Nara received a Bachelor of Engineer in Industrial Engineering from Suranaree University of Technology (2001) and a Ph.D. in Mechatronics from Asia Institute of Technology (2016). His current research interest is how to use simulation techniques and industry 4.0 to develop digital twin systems for the supply chain management. 